\title{
ASPECTOS SINTÁCTICOS DEL DISCURSO NARRATIVO EN EL SIGLO XVII ${ }^{1}$
}

\author{
Elena Olivera Soto \\ Instituto Cervantes de Utrecht \\ elenaoliverasoto@yahoo.es
}

\section{RESUMEN:}

Este artículo se ocupa de los resultados obtenidos con la aplicación de la metodología de análisis sintáctico del profesor Lope Blanch a un corpus de textos narrativos del siglo XVII. Tras estudiar en ellos tanto la extensión como la profundidad de las cláusulas y las relaciones entre estos parámetros, es posible observar las semejanzas y diferencias entre los textos novelescos y los textos no novelescos estudiados.

PALABRAS ClAVE: Sintaxis histórica, «profundidad», análisis sintáctico, textos narrativos.

\section{ABSTRACT:}

This article deals with the results obtained with the application of the syntactic analysis methodology of Professor Lope Blanch in a corpus of narrative texts of the seventeenth century. After studying in both of them the extension and depth of the clauses and the relations between these two parameters, it is possible to observe the similarities and differences between fictional texts and non-fictional texts studied.

KEY WORDS: Historic syntax, «depth», syntactic analysis, narrative texts.

\footnotetext{
${ }^{1}$ Este artículo proviene de un trabajo más amplio, realizado durante el período de investigación del curso «Ampliación de cuestiones en historia de la lengua española» dirigido por D. Francisco Abad Nebot y evaluado ante el tribunal formado por Dña. M ${ }^{\mathrm{a}}$ Luz Gutiérrez Araus, D. Antonio Domínguez Rey y D. Ricardo Maizal Usón, el pasado noviembre de 2010 en la UNED.
} 
La descripción de las unidades sintácticas y, concretamente, de la unidad conocida como oración, es uno de los caballos de batalla de la Gramática a lo largo de la historia. Los distintos enfoques lingüísticos y sus respectivos análisis han creado una gran confusión, no solo conceptual sino también terminológica, en el campo de la sintaxis. Una de las clasificaciones es la realizada por el profesor Juan Miguel Lope Blanch y llevada a la práctica por él mismo en Análisis gramatical del discurso (1987). Basándonos en las novedades que su modelo de análisis proporciona, aplicamos su clasificación de unidades en un corpus de textos narrativos, el cual abarcó diez obras novelescas y diez obras no novelescas de la literatura española del siglo XVII. Nuestro objetivo fue comparar los dos grupos narrativos, para poder establecer las semejanzas y diferencias entre ellos.

Numerosas fueron las variables que estudiamos, pero todas ellas asentadas sobre dos ejes, a los que llamamos 'eje horizontal' y 'eje vertical'.

\section{EJE HoRIZONTAL: LA EXTENSIÓN}

Con 'eje horizontal' nos referimos a la extensión, a la cantidad de elementos que conforman una unidad y la hacen más o menos extensa. Entendimos, siguiendo la clasificación de Lope Blanch, que la expresión autónoma desde el punto de vista elocutivo y con plenitud conceptual respondería a la cláusula, nuestra unidad mayor de análisis. Por ello, el eje horizontal vino determinado por el cotejo de la extensión de unas cláusulas y otras.

Trabajando con corpus lingüísticos y fijando las unidades, siempre nos encontraremos con el obstáculo de la extensión de las mismas. Este parámetro determinará, en ocasiones, diferencias que no son sustanciales para distinguir unos textos de otros, puesto que vienen motivadas por él, salvo el hecho de que hay textos que contienen cláusulas más dilatadas y textos que contienen cláusulas más escuetas.

Si hubiésemos seleccionado cláusulas de aproximadamente la misma longitud y número de subunidades, habríamos estado condicionando nuestros resultados, ya que la muestra habría estado conscientemente escogida, manipulada, y no sería representativa de cada una de las obras. Si, por otro lado, y tal y como hicimos en este trabajo, fijamos un número de unidades superiores — diez cláusulas por obra, sumando en este caso un total de doscientas- contaremos con que habrá textos que contengan componentes más extensos y otros más breves.

El número de apariciones de un determinado ítem en una muestra no es relevante de forma aislada, y para poder compararlo ponderadamente con otras es necesario equilibrar los datos. Por ello, y con el fin de ajustar los resultados simulando lo más posible la situación ideal del análisis, mostramos las cifras en porcentajes cada vez que nos ha sido posible.

Salvado el problema de la extensión para poder establecer nuestras comparativas, solo quedaba pendiente la cuestión de aislar esos datos - a primera vista diferenciadores entre unas muestras y otras- de la extensión misma de las cláusulas. Las ideas que exponemos a lo largo de estas líneas siempre han venido después del contraste de la información de la categoría que estuviésemos observando y de la variable «palabras por cláusula», índice de la longitud de esta. Nuestro fin ha sido, por tanto, separar los fenómenos que son consecuencia de un amplio número de palabras por muestra, de los fenómenos dignos de mención en cuanto originales y determinados por el uso que de la lengua hizo el autor. 
Hechas estas salvedades, adjuntamos los datos extraídos para la comparación y posterior estudio en las siguientes tablas.

\begin{tabular}{|c|l|c|c|c|c|}
\hline \multicolumn{1}{|c|}{ Obra } & Oraciones & $\begin{array}{c}\text { Oraciones } \\
\text { por } \\
\text { cláusula }\end{array}$ & $\begin{array}{c}\text { Palabras } \\
\text { por } \\
\text { oración }\end{array}$ & $\begin{array}{c}\text { Palabras } \\
\text { por } \\
\text { cláusula }\end{array}$ \\
\hline 1 & $\begin{array}{l}\text { La vida del Buscón llamado don } \\
\text { Pablos }\end{array}$ & 25 & 2,5 & 6,2 & 15,5 \\
\hline 2 & Guzmán de Alfarache & 74 & 7,4 & 5,3 & 39,0 \\
\hline 3 & El Quijote & 36 & 3,6 & 8,5 & 30,5 \\
\hline 4 & Historia de Hipólito y Aminta & 53 & 5,3 & 7,1 & 37,6 \\
\hline 5 & El jardín engañoso & 54 & 5,4 & 7,9 & 42,7 \\
\hline 6 & Policisne de Beocia & 55 & 5,5 & 6,8 & 37,6 \\
\hline 7 & El peregrino en su patria & 71 & 7,1 & 7,0 & 49,6 \\
\hline 8 & La peregrinación sabia & 86 & 8,6 & 7,5 & 64,1 \\
\hline 9 & La Galatea & 45 & 4,5 & 7,9 & 35,6 \\
\hline 10 & La constante Amarilis & 39 & 3,9 & 7,9 & 31,0 \\
\hline & \multicolumn{3}{|l|}{} \\
\cline { 2 - 6 } & MEDIA TEXTOS NOVELESCOS & $\mathbf{5 3 , 8}$ & $\mathbf{5 , 3 8}$ & $\mathbf{7 , 2}$ & $\mathbf{3 8 , 3}$ \\
\cline { 2 - 6 } & MEDIA TOTAL & $\mathbf{4 7 , 8}$ & $\mathbf{4 , 7 8}$ & $\mathbf{7 , 3}$ & $\mathbf{3 4 , 1}$ \\
\hline
\end{tabular}

Tabla 1. DATOS DE OBRAS NARRATIVAS NOVELESCAS

\begin{tabular}{|l|l|c|c|c|c|}
\hline \multicolumn{1}{|c|}{ Obra } & Oraciones & $\begin{array}{c}\text { Oraciones } \\
\text { por } \\
\text { cláusula }\end{array}$ & $\begin{array}{c}\text { Palabras } \\
\text { por } \\
\text { oración }\end{array}$ & $\begin{array}{c}\text { Palabras } \\
\text { por } \\
\text { cláusula }\end{array}$ \\
\hline 11 & Providencia de Dios & 70 & 7,0 & 6,3 & 44,1 \\
\hline 12 & El Criticón & 33 & 3,3 & 7,9 & 26,2 \\
\hline 13 & Del Rey y de la Institución Real & 30 & 3,0 & 7,2 & 21,5 \\
\hline 14 & $\begin{array}{l}\text { Historia de las guerras civiles de Gra- } \\
\text { nada }\end{array}$ & 25 & 2,5 & 7,7 & 19,2 \\
\hline 15 & Idea de un príncipe político cristiano & 21 & 2,1 & 6,5 & 13,7 \\
\hline 16 & La Primera Corónica y Buen Gobierno & 31 & 3,1 & 11,3 & 35,0 \\
\hline 17 & Los Comentarios Reales & 55 & 5,5 & 6,1 & 33,7 \\
\hline 18 & Tesoro de la Lengua Castellana & 69 & 6,9 & 6,6 & 45,7 \\
\hline 19 & Historia General de España & 25 & 2,5 & 8,5 & 21,2 \\
\hline \multirow{2}{*}{20} & $\begin{array}{l}\text { Tratado de la religión y virtudes que } \\
\text { debe tener el príncipe cristiano }\end{array}$ & $\mathbf{5 9}$ & 5,9 & 6,6 & 38,8 \\
\hline \multirow{2}{*}{} & \multicolumn{2}{|c|}{25} & & & \\
\cline { 2 - 6 } & MEDIA TEXTOS NO NOVELESCOS & $\mathbf{4 1 , 8}$ & $\mathbf{4 , 1 8}$ & $\mathbf{7 , 5}$ & $\mathbf{2 9 , 9}$ \\
\cline { 2 - 6 } & MEDIA TOTAL & $\mathbf{4 7 , 8}$ & $\mathbf{4 , 7 8}$ & $\mathbf{7 , 3}$ & $\mathbf{3 4 , 1}$ \\
\hline
\end{tabular}

Tabla 2. DATOS DE OBRAS NARRATIVAS NO NOVELESCAS 
De todas estas informaciones, quizá el dato más relevante sea el número de palabras por cláusula, ya que nos permite comparar la longitud de unas cláusulas con otras: como ya apuntamos, a mayor número de palabras, mayor extensión.

En cuanto a la obras novelescas, y siendo su media 38,3 llama especialmente la atención el caso de El Buscón (1) que cuenta tan solo con 15,5 palabras; es decir, esta obra contiene las cláusulas más breves de todas las elegidas.

Quevedo siempre demostró un gran dominio del lenguaje por su habilidad para jugar con él. Su brillante estilo, denominado conceptista, se rige por los siguientes preceptos según Estébanez Calderón (2001: 205):

«El escritor conceptista ofrece al lector la posibilidad de un conocimiento de las cosas, no a través de una descripción directa de las mismas, sino por un haz de relaciones y correspondencias con otros objetos, percibidas en los conceptos creados por él. Un escritor demuestra precisamente su agudeza de ingenio en esa capacidad de establecer relaciones entre los seres para hacerlos más inteligibles. El concepto bien construido provocaría en el lector un efecto similar al del «relámpago en una tempestad nocturna: ilumina con repentina brillantez los objetos que la oscuridad no dejaba distinguir (A. Parker)».

Y precisamente esa conceptualización del lenguaje, esa densidad de información, es la que se pone de manifiesto con nuestras estadísticas. Llama la atención el bajo número de palabras por cláusula - 15,5 - siendo la media de su grupo un 38,3 y la media total de obras de un 34,1, y la también pequeña cifra de palabras por oración: 6,2 en Quevedo, mientras que la media total es de 7,33. Es decir, la prosa de Quevedo se compone de cláusulas breves, estructuradas en oraciones también breves.

Es de señalar en este punto que los cálculos realizados por Lope Blanch a propósito del estilo de Quevedo coinciden con los nuestros:

«Por sí mismo, el hecho de que la oración gramatical propia de la prosa de Quevedo esté constituido solo por unas cinco palabras es sumamente revelador. Es prueba irrefutable de la «densidad» conceptual del estilo de Quevedo: sus oraciones gramaticales están constituidas por los elementos esenciales, sustantivos, con exclusión de los elementos secundarios, matizadores ${ }^{2}$.»

De igual manera, es interesante observar que La peregrinación sabia (8) con 64,1 palabras por cláusula, y El peregrino en su patria (7) con 49,6 palabras por cláusula, destacan por sobrepasar ampliamente la media de su grupo de textos.

Con respecto a las no novelescas, su media se sitúa en 29,9 palabras por cláusula. Son ampliamente superiores en número de palabras Tesoro de la lengua castellana (18), Providencia de Dios (11) y Tratado de la religión... (20), y claramente inferiores Idea de un príncipe... (15) e Historia de las guerras civiles de Granada (14).

Mencionamos aquí la obra que más destaca del grupo, Tesoro de la lengua castellana. El valor de esta obra es indiscutible: no solo es el primer diccionario monolingüe de la lengua española, sino que también es el primero en publicarse en Europa en una lengua vulgar. Pero a diferencia de lo que se piensa hoy día sobre este tipo de manuales, que recogen exclusivamente información lingüística, esta obra proporcio-

${ }^{2 .}$ LOPE BLANCH, J.M. (1987: 174) 
na numerosas informaciones enciclopédicas extralingüísticas. Lo ratificamos con las palabras de Crespo Hidalgo (1992: 113):

«Por todo lo anterior, comprobamos que Covarrubias era plenamente consciente de que no convenía, como principio general de su metodología, elaborar una obra cuya planta fuese la mera acumulación de los muchos datos que tenía a su alcance en su copiosa librería; sino que, por la naturaleza de los lemas contenidos en la nomenclatura, compuesta por $77.89 \%$ de nombres propios, Covarrubias tuvo que incluir las informaciones enciclopédicas esenciales $-\mathrm{y}$, con demasiada frecuencia, también, datos simplemente anecdóticos — para aclarar el 'significado' cultural de este tipo de lemas.»

En el interesante artículo de Crespo Hidalgo oímos hablar de las repetidas ocasiones en las que Covarrubias alude al interés por no extenderse más de lo necesario, con el objetivo de no cansar al lector. No obstante, en numerosas ocasiones encontramos lo contrario ${ }^{3}$.

En la reducida muestra con la que hemos trabajado podemos observar esta dilatación. Si la media total de palabras por cláusula es 34,1 y la media de las obras no novelescas es 29,9 queda patente que la cifra 45,7 del Tesoro es bastante elevada, luego consta de cláusulas extensas.

Es más interesante, sin embargo, comparar todas las obras con la media total, aspecto que podemos apreciar visualmente en el Cuadro 1.

La obra 1 queda muy por debajo de la media total — marcada con una gruesa línea negra- y, sin embargo las obras 7 y 8 - principalmente esta última- rebasan dicho límite considerablemente. Del mismo modo, las obras 11 y 18 superan la línea media, así como las 14 y 15 quedan a gran distancia del límite.

Si estudiamos este gráfico podemos observar que prácticamente todos los textos novelescos analizados rozan el número medio de palabras por cláusula - a excepción de los tres mencionados (texto 1 , texto 7 y texto 8)- No ocurre lo mismo con los textos no novelescos, en los que son solo cuatro los que acarician la media. Llamativo es también el hecho de que los textos novelescos tienden a sobrepasar la cantidad media, mientras que los textos no novelescos se inclinan por utilizar menor cantidad de palabras. Por tanto, concluimos que las cláusulas novelescas son más extensas que las cláusulas no novelescas.

3. CRespo Hidalgo, J. (1992: 111): «Quevedo, con su hiriente gracejo, le colgó al Tesoro el sambenito de obra en la que «el papel es más que la razón», y esta no le falta a Quevedo». 


\section{Número de palabras por cláusula}

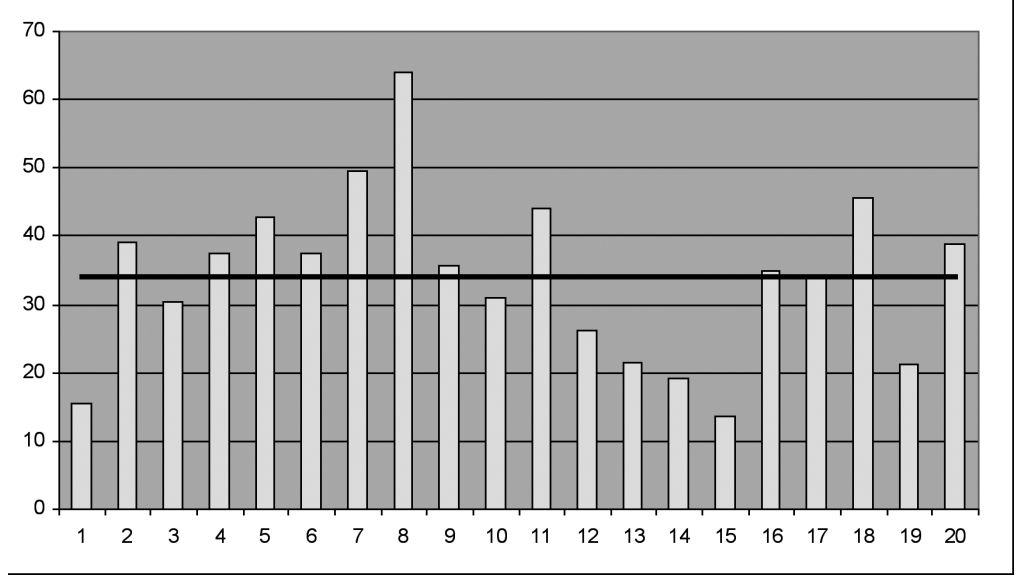

Cuadro 1. PALABRAS POR CLÁUSULA

Ahora bien, hablábamos de dos ejes sobre los que descansaba esta investigación. Tratado el 'eje horizontal' o de la extensión, es el turno ahora del 'eje vertical' o de la profundidad.

\section{EJE VERTICAL: LA PROFUNDIDAD}

La sucesión de unidades en los textos no siempre se produce de forma lineal sino que, a veces, se organiza de manera jerárquica, donde unas unidades contienen a otras, que a su vez contienen a otras, y así sucesivamente. Esta arquitectura sintáctica de los textos vendrá determinada por el número de unidades incluidas en la cláusula y las relaciones entre las mismas. Veamos la definición de cada una de ellas.

El período es la expresión formada por dos o más oraciones o frases entre las que existe una relación sintáctica: coordinada (o paratáctica) o subordinada (o hipotáctica).

El enunciado bimembre en el que existe la predicación - tanto nominal como verbal- y cuyo núcleo es un verbo es nuestra definición de oración. Estas podrán hallarse concatenadas en un período coordinado - y por ende, en el mismo nivel—o en un período subordinado —donde existe una jerarquía y, por ello, organizadas en niveles inferiores respecto a una oración principal-.

La frase es la palabra o conjunto de palabras organizadas en torno a un núcleo y con función identificadora, sin que exista relación predicativa.

De esta manera, una cláusula puede contener períodos, oraciones y/o frases, un periodo contiene oraciones y/o frases, y una oración puede - o no- contener frases. Esta inclusión de unas unidades en otras, formando una ordenación escalonada, muestra lo que llamamos profundidad o verticalidad del texto, y puede observarse esquemáticamente en el Cuadro 2. 


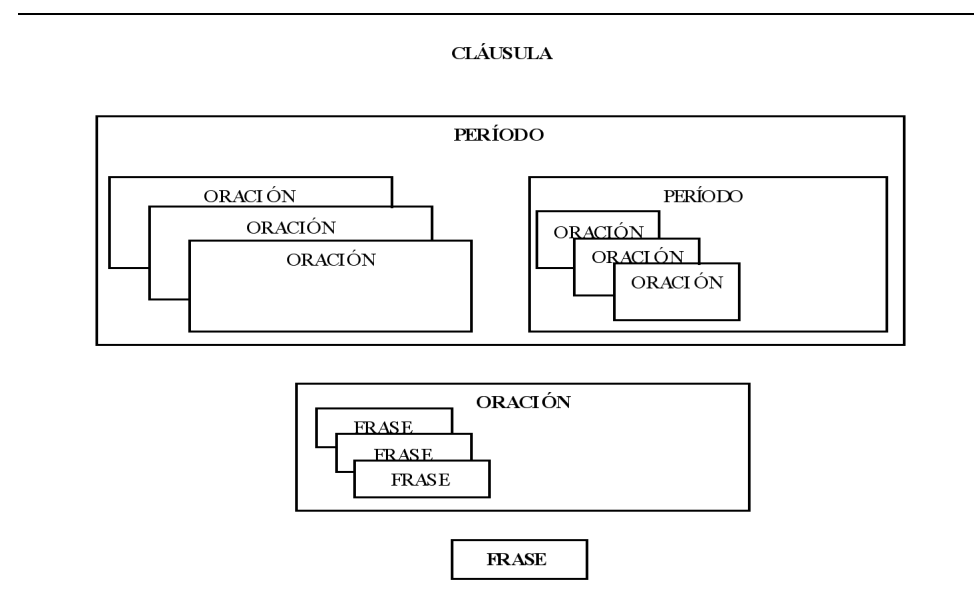

Cuadro 2. ORDENACIÓN JERÁRQUICA DE LAS UNIDADES.

Dicha verticalidad pondrá de manifiesto no solo el número de unidades que conforma cada cláusula, sino las relaciones de dependencia entre estas, dibujando así su estructura interna.

Nos parece relevante confrontar el nivel máximo de profundidad medio de cada obra; esto es, la media de los niveles máximos que alcanza cada cláusula en cada obra. Una vez más, marcamos con una línea la media del total de muestras estudiadas.

\section{Nivel de profundidad}

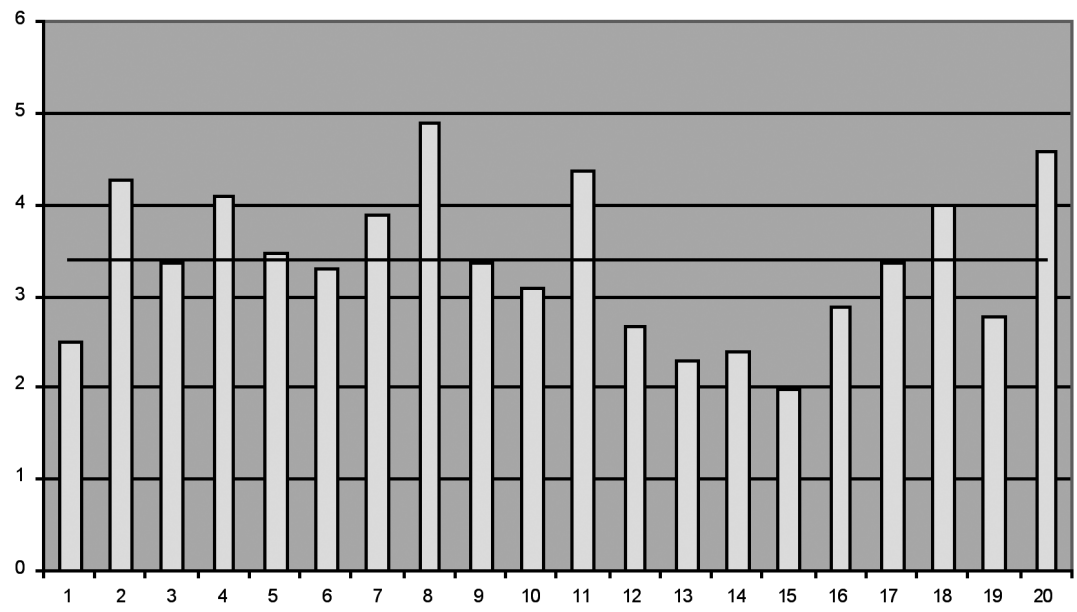

Cuadro 3. NIVEL DE PROFUNDIDAD. 
En los textos novelescos, son ocho las obras que están en la media o la superan, y podemos afirmar que todos ellos tienen unidades que alcanzan, de media, el tercer nivel de profundidad (exceptuando el caso de El Buscón). Confirmamos, pues, que es un grupo homogéneo en cuanto a este parámetro.

En los textos no novelescos no sucede igual, ya que la mayor parte no llega a la media - e incluso la mitad de ellos no alcanza el tercer nivel del que hablábamos con los textos novelescos-; sin embargo, las obras 11, 17, 18 y 20 aparecen con un comportamiento diferente al de su grupo superando el límite del que estamos hablando. Interesante dato, ya que son las mismas obras que también diferían de las de su grupo en cuanto a la extensión.

Está claro que el nivel de profundidad del que hablamos está íntimamente relacionado con el número de subordinación con que cuente el texto. Así lo podemos ver en la siguiente gráfica:

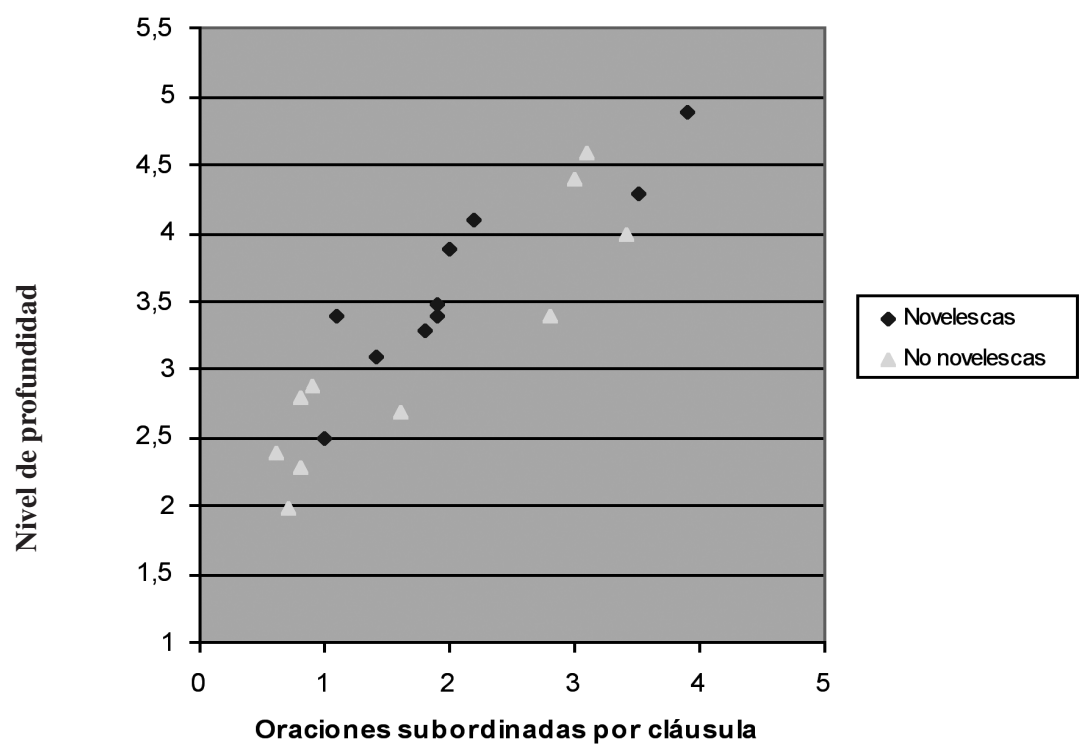

\section{Cuadro 4. PROFUNDIDAD Y ORACIONES SUBORDINADAS}

A mayor número de oraciones subordinadas, mayor profundidad; de ahí la progresión lineal que podemos advertir en el Cuadro 4. Podríamos establecer un patrón diferente para obras novelescas y no novelescas por la posición de las mismas en el gráfico; en él se prueba que las obras novelescas son más profundas que las no novelescas - lo que supone, en contraposición, un mayor número de oraciones simples y/o coordinadas en el grupo de las no novelescas - a excepción de cuatro muestras no novelescas, que fueron mencionadas anteriormente, ya que actuaban de forma anómala con respecto a su grupo cuando hablamos de la extensión, y que corresponden a las obras $11,17,18$ y 20 .

Ahora bien, y aun corroborando que los textos novelescos cuentan con mayor profundidad y por tanto, con mayor subordinación, hay una distinción más, también 
observable a través del análisis, con respecto al tipo de subordinación más abundante en cada uno de los grupos. Puesto que los textos novelescos narran una historia de ficción que debe ser necesariamente situada en unas circunstancias de tiempo, modo, lugar, causa... las más numerosas serán las subordinadas adverbiales. Por el contrario, los textos no novelescos contarán con mayor número de subordinación sustantiva, imprescindible en los textos de carácter expositivo.

\section{Frecuencia total por tipo de oración subordinada}
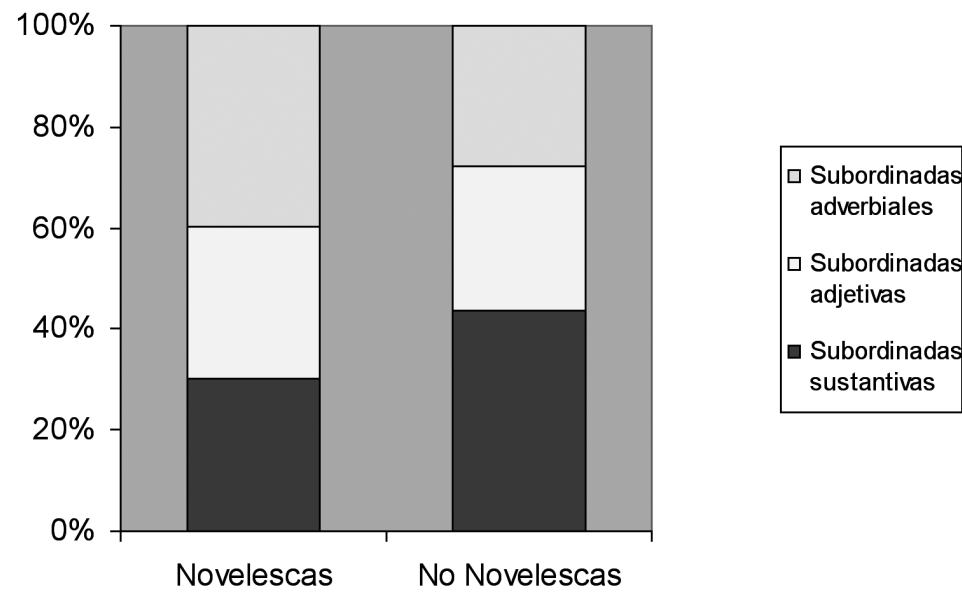

Cuadro 5. FRECUENCIA DE ORACIONES SUBORDINADAS

Un dato más podría ser puesto en relación con los períodos y oraciones subordinados: los gerundios abundan más en los textos novelescos y los infinitivos en los textos no novelescos. Los gerundios — de naturaleza verbal-adverbial— son más frecuentes en el grupo donde la subordinación adverbial es más notable, y los infinitivos - de naturaleza verbal-nominal- son más habituales en el conjunto en el que abunda la subordinación sustantiva.

Los participios son los que más aparecen, con un $47 \%$ en los textos novelescos, como muestra del ornamento lingüístico y de la connotación emotiva de los textos propiamente literarios. Los infinitivos - un $42 \%$ - abundan en los textos no novelescos, como muestra de la conceptualización de la realidad que se expone en ellos.

\section{RELACIÓN ENTRE LOS EJES: EXTENSIÓN Y PROFUNDIDAD}

Si relacionamos ambos ejes, el horizontal o de la extensión con el vertical o de la profundidad, los resultados que obtendremos son los que siguen.

La longitud de los textos está estrechamente relacionada con la profundidad de las mismas, extrayendo dos consecuencias: a) a mayor número de oraciones, mayor profundidad, y b) mayor profundidad solo en oraciones breves.

Representando cada cláusula en el Cuadro 6, es clara la relación entre ambos ejes: con respecto a la extensión, podemos verificar la media de 7 palabras de media por 
oración; con respecto a la profundidad ${ }^{4}$, decidimos otorgar un valor a cada cláusula que fuera mayor que la media de profundidad máxima de la que hablamos, con el fin de contar con un espectro más amplio para la gráfica.

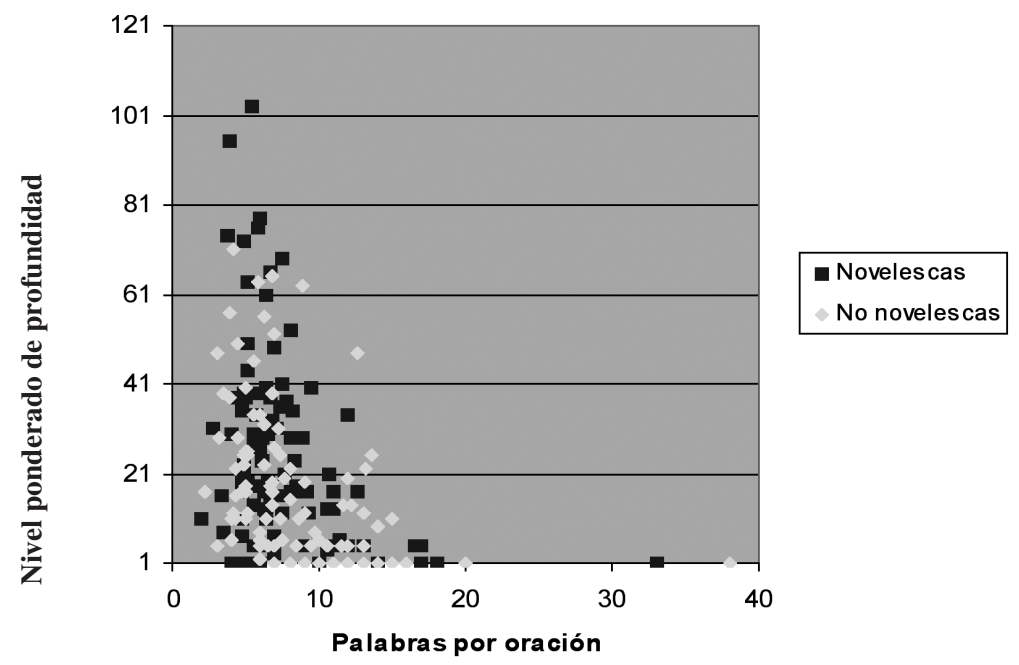

Cuadro 6. PROFUNDIDAD Y PALABRAS POR ORACIÓN

Cuando contamos con una elevada cifra de palabras por oración, el resultado con respecto a la profundidad siempre es bajo. Pero cuando contamos con oraciones breves, el nivel de profundidad puede variar. O dicho al contrario: para que se den niveles altos de profundidad es indispensable que las oraciones sean breves — nótese que no hay ni uno solo de nuestros ejemplos en la parte superior derecha, la reservada para oraciones extensas y profundas-. Es también muy sencillo constatar que los textos novelescos tienen mayor nivel de profundidad que los no novelescos, como expusimos líneas atrás.

No nos aventuraremos a establecer otras coincidencias o diferencias entre los textos, ni a extender lo visto aquí como caracterizador de la amplia narrativa que compone el siglo XVII. Para ello habría que contar con una muestra mucho mayor y, por ende, más representativa. Del mismo modo, asegurar unos rasgos como propios de una época o de un género determinado, sería más fácil si ampliáramos el corpus a otras épocas o géneros literarios con los que establecer la comparación. Ambas son interesantes líneas de trabajo que quedan abiertas a estudios posteriores y que no tienen cabida entre estas páginas.

\footnotetext{
${ }^{4}$ Hemos dado un valor a cada nivel de profundidad: 1 para el primer nivel, 2 para el segundo nivel, 3 para el tercer nivel, y así sucesivamente, siendo un valor mayor el nivel más profundo. En cada uno de los niveles puede haber una o varias unidades, y a cada una de ellas se les ha otorgado el valor antes fijado, teniendo un valor total por nivel resultante de la suma de unidades en el mismo. Sumando los valores de cada nivel obtendríamos el que hemos llamado nivel ponderado de profundidad de cada cláusula.
} 


\section{BIBLIOGRAFÍA:}

AAVV (2004) Disquisiciones sobre filología hispánica. In memoriam Juan M. Lope Blanch (Gloria Báez y Elisabeth Luna coord.) Universidad Nacional Autónoma de México.

ABAD NeBOT, F. (1975) «Las unidades como problema de lógica de la investigación lingüística», en Revista Española de Lingüística (págs. 139-148) en www.uned.es/sel/pdf/ene-jun75/5,1\%20Abad.pdf

- (2008) Historia general de la lengua española. Tirant lo Blanch.

ANRUBiA, E. (2002) «La estructura narrativa del ser humano», en Espéculo. Revista de estudios literarios. Universidad Complutense de Madrid.

Crespo Hidalgo, J. (1992) «El arte diccionarista de Sebastián de Covarrubias», en E.L.U.A. 8, 1992 (págs. 99-132), en http://rua.ua.es/dspace/bitstream/10045/6483/1/ELUA_08_06.pdf

EstéBANEZ CALDERÓN, D. $\left(2001^{2}\right)$ Diccionario de términos literarios. Alianza.

GutiérRez Araus, M. L. (1993) Estructuras sintácticas del español actual. Sociedad General Española de Librería.

GuTIÉRREZ ORDÓÑEZ, S. (1984) «¿Es necesario el concepto «oración»?» en Revista Española de Lingüística, 14 (págs. 245-270)

- (1997) Principios de sintaxis funcional. Arco-Libros.

LAPESA, R. (19819) Historia de la lengua española. Gredos.

LoPe Blanch, J.M. (1979) Concepto de oración en la lingüística española. Universidad Nacional Autónoma de México.

— (1980) «La estructura de la cláusula en dos obras medievales», en AIH. Actas VII.

- $\left(1987^{2}\right)$ Análisis gramatical del discurso. Universidad Nacional Autónoma de México.

- (1992) Nuevos estudios de Lingüística hispánica. Universidad Nacional Autónoma de México.

Martínez, M. (2000) «Dualismo metafísico e inmortalidad del alma en Providencia de Dios de Francisco de Quevedo», en Espéculo. Revista de estudios literarios. Universidad Complutense de Madrid, en http://www.ucm.es/info/especulo/numero16/dualismo.html

MARTínez LinARES, M. A. (1989) «La «oración compuesta por subordinación» en la gramática de Eduardo Benot» en http://rua.ua.es/dspace/bitstream/10045/6595/1/ELUA_05_13.pdf

MÉNDEZ GarCía De PAREDES, E. (1993) «Reflexiones sobre la oración compleja en español» en http://www.uned.es/sel/pdf/jul-dic-93/23-2-Mendez.pdf

MolL, J. (2008) «La narrativa castellana a comienzos del siglo XVII: aspectos editoriales», en Anales Cervantinos, XL (págs. 31-46)

Montes GiRALDo, J.J. (1986) «Frase nominal, frase verbal y enunciados impersonales», en http://cvc.cervantes.es/lengua/thesaurus/pdf/41/TH_41_123_327_0.pdf

NARBona JimÉnez, A. (1989) Sintaxis española: nuevos y viejos enfoques. Ariel Lingüística.

Osuna GARCíA, F. (2006) «De nuevo sobre el concepto de oración», en http://elies.rediris.es/Language_Design/LD8/1-Osuna-LD8.pdf

Pedraza Jiménez, F.B.; Rodríguez CÁCeres, M. $\left(2007^{3}\right)$ Las épocas de la literatura española. Ariel.

Penny, R. (2006²) Gramática histórica del español. Ariel.

R.A.E. $\left(2006^{23}\right)$ Esbozo de una nueva gramática de la lengua española. Espasa.

Rojo, G. (1978) Cláusulas y oraciones. Universidad de Santiago de Compostela.

- (1983) Aspectos básicos de sintaxis funcional. Ágora. 
